\title{
Neuropsychological profiles and cerebral glucose metabolism in neurocognitive Long COVID-syndrome
}

Andrea Dressing ${ }^{1,2 *}$, Tobias Bormann ${ }^{1,2 *}$, Ganna Blazhenets ${ }^{3}$, Nils Schroeter ${ }^{1}$, Lea I. Walter ${ }^{1}$, Johannes Thurow ${ }^{3}$, Dietrich August ${ }^{4}$, Hanna Hilger ${ }^{4}$, Katarina Stete ${ }^{4}$, Kathrin Gerstacker $^{5}$, Susan Arndt ${ }^{5}$, Alexander Rau ${ }^{6}$, Horst Urbach ${ }^{6}$, Siegbert Rieg ${ }^{4}$, Dirk Wagner ${ }^{4}$, Cornelius Weiller ${ }^{1,2}$, Philipp T. Meyer ${ }^{3 *}$, Jonas A. Hosp ${ }^{1 *}$

1: Department of Neurology and Clinical Neuroscience, Medical Center - University of Freiburg, Faculty of Medicine, University of Freiburg, Freiburg, Germany

2: Freiburg Brain Imaging Center, Medical Center - University of Freiburg, Faculty of Medicine, University of Freiburg, Freiburg, Germany

3: Department of Nuclear Medicine, Medical Center - University of Freiburg, Faculty of Medicine, University of Freiburg, Freiburg, Germany

4: Division of Infectious Diseases, Department of Medicine II, Medical Center - University of Freiburg, Faculty of Medicine, University of Freiburg, Freiburg, Germany

5: Department of Otorhinolaryngology - Head and Neck Surgery, Medical Center - University of Freiburg, Faculty of Medicine, University of Freiburg

6: Department of Neuroradiology, Medical Center - University of Freiburg, Faculty of Medicine, University of Freiburg, Freiburg, Germany

* Equal contribution

Corresponding author:

Jonas A. Hosp MD

E-mail: jonas.hosp@uniklinik-freiburg.de

University Medical Center Freiburg

Department of Neurology and Neuroscience

Breisacher Str. 64, 79106 Freiburg, Germany

phone:+49 76127050010

\section{First author:}

Andrea Dressing MD

E-mail: andrea.dressing@uniklinik-freiburg.de

University Medical Center Freiburg

Department of Neurology and Neuroscience

Breisacher Str. 64, 79106 Freiburg, Germany

phone:+49 76127050010

Running head: Cognition and ${ }^{18}$ F-FDG PET in Long COVID 
Immediate Open Access: Creative Commons Attribution 4.0 International License (CC BY) allows users to share and adapt with attribution, excluding materials credited to previous publications.

License: https://creativecommons.org/licenses/by/4.0/. Details: https://inm.snmjournals.org/page/permissions.

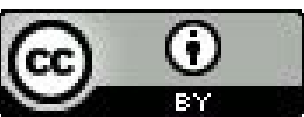




\section{ABSTRACT}

During the Corona Virus Disease 2019 (COVID-19) pandemic, Long COVID-syndrome, which impairs patients through cognitive deficits, fatigue, and exhaustion, has become increasingly relevant. Its underlying pathophysiology, however, is unknown. In this study, we assessed cognitive profiles and regional cerebral glucose metabolism as a biomarker of neuronal function in outpatients suffering from long-term neurocognitive symptoms after COVID-19.

Methods: Outpatients seeking neurological counseling with neurocognitive symptoms persisting for more than three months after polymerase chain reaction (PCR)-confirmed COVID-19 were included prospectively between June 16, 2020, and January 29, 2021. Patients ( $n=31,54 \pm 2.0$ years) in the long-term phase after COVID-19 (202 \pm 58 days after positive PCR) were assessed with a neuropsychological test battery. Cerebral ${ }^{18} \mathrm{~F}$-FDG PET imaging was performed in 14/31 patients.

Results: Patients self-reported impaired attention, memory, and multitasking abilities (31/31), word-finding difficulties (27/31), and fatigue (24/31). Twelve of 31 patients could not return to the previous level of independence/employment. For all cognitive domains, average group results of the neuropsychological test-battery showed no impairment, but deficits $(z$-score $<-1.5)$ were present on single-patient level mainly in the domain of visual memory (in $7 / 31$; other domains $\leq 2 / 31)$. Mean MoCA performance (27/30 points) was above the cutoff-value for detection of cognitive impairment ( $<26$ points), although $9 / 31$ patients performed slightly below this level (23-25 points). In the subgroup of patients who underwent ${ }^{18} \mathrm{~F}$-FDG PET, we found no significant changes of regional cerebral glucose metabolism. 
Conclusions: Long COVID patients self-report uniform symptoms hampering their ability to work in a relevant fraction. However, cognitive testing showed minor impairments only on single-patient level approximately six months after the infection, whereas functional imaging revealed no distinct pathological changes. This clearly deviates from previous findings in subacute COVID-19 patients, suggesting that underlying neuronal causes are different and possibly related to the high prevalence of fatigue.

Keywords: Long COVID-syndrome, cognition, fatigue, ${ }^{18} \mathrm{~F}-\mathrm{FDG}$ PET, Montreal Cognitive Assessment 


\section{INTRODUCTION}

As the Corona Virus Disease 2019 (COVID-19) pandemic proceeds, the long-term consequences like chronic neurocognitive symptoms after infection with the severe acute respiratory syndrome coronavirus-2 (SARS-CoV-2) are an increasingly recognized problem. A multitude of previously healthy patients self-report symptoms like 'brain-fog', memory loss (18-40\%), attentional problems (16-34\%), and fatigue $(60-70 \%)$ months after the acute infection has long subsided (1-6). The label Long COVID-syndrome has recently been established for these symptoms in the aftermath of an acute SARS-CoV-2 infection (7), however, the underlying pathophysiology remains unclear.

We described impaired cognitive functions associated with frontoparietal hypometabolism (indicating cortical dysfunction) on ${ }^{18}$ F-FDG PET (8) in COVID-19 patients approximately one month after the acute infection. Using voxel-wise principal components analysis, a COVID-19-related spatial covariance pattern has emerged, the expression of which tightly correlated with performance in the Montreal Cognitive Assessment (MoCA). In a subgroup of these patients, a long-term follow-up (approx. 6-7 months after infection) revealed a substantial, but still incomplete recovery of cognitive deficits and cortical dysfunction on ${ }^{18} \mathrm{~F}-\mathrm{FDG}$ PET (9). Likewise, a predominantly frontal cortical hypometabolism, which improved during follow-up after six months, was detected in patients suffering from COVID-19-related encephalopathy (10). Deviating from these findings, regional hypometabolism of limbic/paralimbic regions extending to the brainstem and cerebellum (11) or hypometabolism of the right parahippocampal gyrus and thalamus (12) has been described in COVID-19 patients examined at three to four months after symptom onset. 
Postmortem neuropathological examinations in COVID-19 patients revealed pronounced glial activation and infiltration by cytotoxic $\mathrm{T}$ lymphocytes in brainstem and cerebellum (13), which is likely caused by a systemic inflammatory response or a cytokine release (14). Since the cortical grey matter is largely unaffected by inflammatory changes $(8,13)$, reduction of cortical glucose metabolism in early subacute patients $(8-10)$ might be caused by a functional decoupling from afferents, which is in line with recovery of cognitive deficits and cortical metabolism in long-term follow-up investigations $(9,10,15)$.

Thus, the question arises whether alterations in cerebral glucose metabolism are also present in patients with Long COVID-syndrome as a potential pathophysiological correlate of the neurocognitive symptoms. We present data from a prospective cohort of outpatients about six months after SARS-CoV-2 infection who self-reported persistent subjective neurocognitive symptoms. Cognitive performance and cerebral ${ }^{18} \mathrm{~F}-\mathrm{FDG}$ PET were assessed to objectify subjective symptoms and to investigate possible similarities to previously observed changes in early subacute patients. 


\section{MATERIALS AND METHODS}

\section{Participants}

We report data from a monocentric, prospective cohort of 31 patients (age $53.6 \pm 12.0$ years; $11 / 20$ male/female) who were admitted to the outpatient clinic of the Department of Neurology and Clinical Neuroscience of the University Hospital Freiburg between June 16, 2020, and January 29, 2021 due to lasting neurocognitive symptoms in the chronic phase (>3 months) after COVID-19. Inclusion criteria were: 1 ) history of reverse transcription polymerase chain reaction (rt-PCR)-confirmed SARS-CoV-2 infection; 2) presence of new subjective neurocognitive symptoms persisting for longer than three months after positive rt-PCR; 3 ) age $>18$ years. Exclusion criteria were any preexisting neurodegenerative disorders. One patient refused to participate. See Supplement Table 1 for detailed demographic data.

Importantly, the current "Long COVID-cohort" shares no overlap with previous studies on COVID-19 from our group (8,9). In these, subacute inpatients (3-4 weeks post COVID) were screened independently from subjective complaints and included if they met inclusion criteria (most importantly, at least two new neurological symptoms to qualify for PET) (8). A subset of eight patients were furthermore eligible for a follow-up (9). In contrast, the present cohort results from self-referral because of new neurocognitive symptoms, which may not necessarily be verified by further examinations (see below).

The present study was approved by the local ethics committee of the University Medical Center Freiburg (EK 211/20) and complies with the Helsinki Declaration of 1975, as revised in 2008. Written informed consent was obtained from all patients. 


\section{General examination}

General neurological deficits were examined in a complete neurological assessment by a board-certified neurologist ( $>5$ years of training). The degree of actual disability was graded as follows: 0 , no relevant restrictions; 1 , relevant restrictions but able to work; 2, reduction of work quota necessary; 3 , inability to work and/or restriction of daily life activities. Disease severity during the acute stage was scored according to a modified version of the German definitions (16): 1, no signs of pneumonia; 2, pneumonia, outpatient treatment; 3, pneumonia, inpatient treatment; 4, ARDS, endotracheal ventilation at ICU. A subgroup of six patients received structural MRI (Supplement Methods).

\section{Cognitive functions}

All patients were examined with a 50-min cognitive battery administered in German (native) language in a constant order by a trained neuropsychologist. The neuropsychological test battery comprised Hopkins Verbal Learning Test-Revised (HVLT (17)), Brief Visuospatial Memory Test-Revised (BVMT-R (18)), Digit Span forward/reverse (19), Trail Making Test part A/B (20), Color-Word Interference Test (FWIT (21), Symbol-Digit Modalities Test (SDMT (22)), and a semantic and letter fluency test (23). Individual raw scores were z-transformed based on the normative sample as reported in the manuals. Results were stratified by age and education where available. In the case of the FWIT, raw scores were assigned a T-score, which then was transformed

into a z-score. Z-scores for each domain and a composite z-score which represents overall cognitive functions of the patients were calculated by averaging the $z$-sores based 
on Lazar et al. (24) with minor adjustments. The threshold for impaired performance was defined as 1.5 SD below the normative mean (24). Additionally, the MoCA (version 7.1, www.mocatest.org (25)) was applied (maximum achievable score $=30$, higher scores indicating better performance). The cutoff score for cognitive impairment was defined as performance below 26 (25). Correction for years of education $(\mathrm{YoE})$ was performed $(+1$ point in case of $\leq 12 \mathrm{YoE}$ ). Fatigue was assessed using the Würzburg Fatigue Inventory in Multiple Sclerosis (WEIMuS (26)), a self-rating questionnaire for symptoms of physical and cognitive fatigue. In addition, the Geriatric Depression Scale-15 (GDS (27)) was included. Scores for MoCA, Fatigue and the GDS were not included in the composite score.

\section{${ }^{18}$ F-FDG-PET Imaging}

Cerebral ${ }^{18} \mathrm{~F}-\mathrm{FDG}$ PET was recommended to all patients based on clinical indication for diagnosis of persistent unexplained cognitive impairment (including the exclusion of other causes) based on previous reports on altered cerebral glucose metabolism in COVID-19 patients $(8,10-12)$ and performed in 14/31 patients on average $197.9 \pm 61.1$ days after manifestation of COVID-19 as indicated by the first positive PCR. PET scans (10-min duration) were acquired on a fully digital Vereos PET/CT scanner (Philips Healthcare, The Netherlands) 50 minutes after intravenous injection of $211 \pm 9$ $\mathrm{MBq}{ }^{18} \mathrm{~F}-\mathrm{FDG}$ under euglycemic conditions at rest (eyes open, reduced ambient noise). All individual scans were read as part of the clinical routine by two experienced Nuclear Medicine physicians (>20 and 5 years of experience in brain PET) using highly standardized displays of 30 transaxial ${ }^{18} \mathrm{~F}-\mathrm{FDG}$ PET slices (hot metal color scale; maximum and minimum thresholds set to 1.8 [100\%] and 0.09 [5\%], respectively, after 
voxel-wise data normalization to mean uptake in brain parenchyma) and voxel-based statistical analyses using three-dimensional stereotactic surface projections (3DSSP/Neurostat (28)) and appropriate age-matched controls.

Group analyses were performed as previously described (8): After spatial normalization and smoothing (isotropic Gaussian kernel, $10 \mathrm{~mm}$ full width at half maximum), the pattern expression score (PES) of the previously established COVID-19related spatial covariance pattern was derived by the topographic profile rating algorithm, reflecting the expression of the established pattern in each individual's data. For statistical comparison, we also assessed the PES of the COVID-19-related covariance pattern in control patients ( $n=45$; age $63.0 \pm 9.1$ years; range: $50-85$ years; $27 / 18$ male/female) scanned under identical conditions (for details see (8)). As confirmatory analysis, a voxelwise analysis of covariance (ANCOVA) with age and sex as covariates was calculated with Statistical Parametric Mapping (SPM) for comparison of Long COVID and control patients. For count rate normalization we employed proportional scaling of each individual's ${ }^{18} \mathrm{~F}-\mathrm{FDG}$ PET to the mean uptake in a brain parenchyma mask (SPM tissue probability map, white and grey matter probability $>50 \%$ excluding CSF with probability $>30 \%$ ). A false discovery rate (FDR) corrected $P<0.05$ was used as a statistical threshold. The correlation between mean z-scores of the domains (attention, executive function, processing speed, verbal and visual memory), composite z-score, MoCA, WEIMuS cognitive and physical fatigue scores and voxelwise ${ }^{18}$ F-FDG uptake was analyzed by SPM-based regression analyses. FDR-corrected $P<0.05$ and uncorrected $P<0.005$ (cluster size $>30$ voxels) were used as statistical thresholds. All processing steps were implemented with an in-house pipeline using MATLAB (The MathWorks, Inc., Natick, 
Massachusetts, United States) and SPM (SPM12; The Wellcome Centre for Human Neuroimaging, UCL Queen Square Institute of Neurology, London, UK) software.

\section{Statistical analysis}

Statistical analyses were performed using IBM SPSS Statistics version 27 (Ehningen, Germany) and R (https://www.R-project.org/). Shapiro-Wilk- and KolmogorovSmirnov-tests were used to confirm normal distribution. Correlations between demographic/clinical data and neurocognitive test scores were exploratorily assessed with Spearman's rank correlation test. For group comparisons of neurocognitive test scores one sample $t$ - or Mann-Whitney-U-tests were performed. Group difference of the PES of Long COVID patients and control patients was tested with an ANCOVA including age and sex as covariates. The strength of the relationship between the PES of the COVID-19-related covariance pattern and the results from cognitive assessments was estimated with Spearman's rank partial correlation test adjusted for the patient's age. 


\section{RESULTS}

The neurological examination (202.3 \pm 57.5 days after first positive COVID-19PCR) revealed no focal deficit related to SARS-CoV-2 infection. Contrary, all patients complained about difficulties in attention, memory, and multitasking abilities. Moreover, $24 / 31(77 \%)$ complained about fatigue. Three of $31(10 \%)$ patients reduced their work quota due to these symptoms; $9 / 31$ (29\%) patients were unable to work and/or restricted their activities of daily living at the time of examination. Actual disability was significantly correlated with severity of initial disease $(R=0.38 ; P=0.03)$. Basic clinical data are summarized in Supplement Table 2.

Six of 31 patients underwent cerebral MRI (four with contrast enhancement). Upon visual assessment, microembolic subacute cortical infarction was observed in the left occipital lobe in one patient (65-year-old male), and slight microangiopathic changes corresponding to Fazekas 2 were present in a 61-year-old female. No other structural changes, and in particular no sign of atrophy, acute encephalitis or leptomeningeal enhancement, were found.

\section{Cognitive functions}

The mean z-scores of verbal and visual memory domains and composite z-score were not significantly different from zero (all $P>0.1$ ). The mean $z$-scores for executive functions $(P<0.05)$, attention $(P<0.01)$, and processing speed $(P<0.01)$ were even higher than zero and, in total, almost half of the patients $(n=15,49 \%)$ were completely unimpaired in the neurocognitive test battery (Supplement Table 3). However, some 
patients exhibited mild to moderate impairments in single domains: the most frequently impaired domain was visual memory (7/31 [23\%] patients; other domains $\leq 2 / 31[\leq 7 \%])$. Impaired individual tests on single-subjects level were most frequently observed for verbal and visual memory tests (number of impaired patients 3-7 [10-23\%] and 4-8 [13-26\%], respectively; Supplement Table 3).

Although the mean group MoCA performance $(26.6 \pm 2.2$ points) was above the cutoff (25), mild impairment was detected in nine patients (29\%; range $23-25$ points). The greatest variance was observed for the recall task of the MoCA $(3.2 \pm 1.6$ points, $16 / 31$ patients scoring below 4 points). Of note, the group of patients with impaired MoCA test did not differ in terms of age or delay between infection and examination from the rest of the cohort (both $P>0.5)$.

Upon a self-rating questionnaire $61 \%(n=19)$ revealed overall symptoms of fatigue. On subscore level $67 \%(n=21)$ were above the cutoff for cognitive fatigue and $42 \%(n=13)$ above the cutoff for physical fatigue (Supplement Table 4). The GDS $(3.9 \pm 2.6)$ indicated no relevant level of depression in the present patient cohort; only four individuals slightly exceeded the cut-off value (range 8-10 points) indicating mild depressive symptoms (27) (Supplement Table 4).

Performance on MoCA was correlated with the composite z-score of the neurocognitive test battery $(R=0.53 ; P<0.05)$. In turn, self-rated fatigue (WEIMuS sum score) correlated significantly with self-rated depression (GDS, albeit in a subclinical range; $\mathrm{R}=0.61 ; P<0.001)$. MoCA test scores and the overall composite $z$-score did not correlate with self-rated depression or any aspect of self-rated fatigue. Except for positive correlations of initial disease severity with physical fatigue $(\mathrm{R}=0.37 ; P<0.05)$, clinical 
parameters like disease severity and degree of actual disability did not correlate with performance on cognitive and other tests.

\section{${ }^{18}$ F-FDG PET Imaging}

Patients undergoing ${ }^{18} \mathrm{~F}-\mathrm{FDG}$ PET did not differ from those who did not in terms of epidemiological variables (age, sex) or results of the neuropsychological test battery (all P>0.05, Supplement Table 1-4). Clinical routine assessments of each patient's ${ }^{18} \mathrm{~F}-\mathrm{FDG}$ PET scan revealed no distinct pathological findings (Figure 1). In particular, none of the patients exhibited a frontoparietal predominant hypometabolic pattern previously described in subacute COVID-19 inpatients (8). Likewise, PET scans suggested no alternative diagnoses (e.g., encephalitis, neurodegenerative dementia) in any case.

Group averaged ${ }^{18} \mathrm{~F}$-FDG PET scans in Long COVID and control patients are shown in Figure 2. None of the patients expressed the previously established COVID-19-related spatial covariance pattern, with individual PES ranging from -7 to -60 . There was no significant group difference in PES between Long COVID patients $(-36.7 \pm 17.3)$ and control patients $(-11.3 \pm 29.2)$ after adjustment for age and sex (ANCOVA, factor group: $P=0.14)$.

In contrast to our previous studies in COVID-19 inpatients with novel neurological symptoms in the subacute stage (8) and at follow-up (9), no significant relationship between MoCA and PES was found $(\mathrm{R}=-0.17, P>0.5)$. There was also no significant correlation of PES with fatigue, composite or domains $z$-scores (all $P>0.5$ ). Confirmatory voxel-wise SPM analyses yielded no regions with significantly (FDR-corrected $P<0.05$ ) 
different glucose metabolism (neither hyper- nor hypometabolism) in Long COVID patients compared to control patients. Moreover, no association to MoCA, domain zscores, composite z-score, or WEIMuS fatigue scores were found by voxel-wise regression analyses (FDR-corrected $P<0.05$ ). No relevant findings were observed at exploratory statistical threshold of uncorrected $P<0.005$. 


\section{DISCUSSION}

Present study reports a prospective assessment of 31 patients self-presenting to our outpatient clinic because of neurocognitive symptoms more than 6 months after a SARS-CoV-2 infection with Long COVID-syndrome. Although 39\% of patients report a relevant disability at work and every-day life due to these symptoms, an exhaustive assessment including a detailed cognitive battery showed only mild impairment in individual patients and cerebral ${ }^{18} \mathrm{~F}-\mathrm{FDG}$ PET failed to reveal a distinct pathological signature.

Cognitive profiles in our sample revealed an overall normal to higher-than-normal performance in all cognitive domains (verbal memory, visual memory, processing speed, attention, executive function) and on MoCA (average score 27/30, cutoff value for detection of any cognitive impairment $<26 / 30(25))$. However, impairments were present on a single-patient level, especially in the domain of visual memory (Supplement Table 3). Furthermore, 9/31 (29\%) patients performed below the MoCA cutoff value indicating mild cognitive impairments.

These results indicate that in some patients with Long COVID, discrete neurocognitive impairments may be present, which is in line with findings from other studies: deficits in verbal/visual memory, executive functions, verbal fluency, attention, and language were reported six to nine months after the infection, which were correlated in their expression with the initial degree of severity (29). Another study reported mild deficits in episodic memory function (up to 6 months after the infection), and vigilance and motivation deficits (up to 9 months after the infection); the deficits normalized after the corresponding period of time (15). This, in combination with the longitudinal assessment 
of COVID-19 patients from our group $(8,9)$ suggests that the cognitive deficits are subject to a dynamic process, which might also explain why most patients are cognitively unimpaired in the present long-term study.

It has to be noticed that a rather liberal threshold for definition of impaired cognition was employed ( $1.5 \mathrm{SD}<$ normative mean). This threshold corresponds to a one-sided $p$ value of about 0.07 , which increases the risk of false-positive results and is only slightly below the frequency of impaired observations on detailed tests (see Supplemental Table 3: $45 / 628,6.6 \%)$. That impaired scales accumulated in verbal and visual memory tests is not surprising, as the HVLTR and BVMT are especially challenging and susceptible for attentional fluctuations $(24,28)$. Such fluctuations may also explain why more patients showed impairments in the recognition (i.e., 8/31) when compared to the delayed recall part (i.e., 4/31) of the BVMT - although the latter usually detects deficits with a higher sensitivity (30). Although the comprehensive neuropsychological test battery indicated slight deficits at the level of individual patients, affection of MoCA-performance seemed to be more severely pronounced. This could also be explained by motivational deficits, attentional fluctuations and exhaustion as the MoCA was performed at the end of the test battery.

Previous studies in subacute COVID-19 patients showed deficits in executive and attentional functions, memory, and visuospatial functions that point to a cortical dysfunction with a frontoparietal emphasis $(4,29,31)$. As a correlate of impaired cognitive functions, we recently described a predominantly frontoparietal cortical hypometabolism on ${ }^{18} \mathrm{~F}$-FDG PET in subacute COVID-19 patients $(8,9)$. Thus, we also performed ${ }^{18} \mathrm{~F}-\mathrm{FDG}$ PET in the present sample of patients with neurocognitive Long COVID-syndrome to 
objectify changes of regional neuronal function by an approach that is independent of the patients' test compliance and can be analyzed complete observer-independent. Individual ${ }^{18}$ F-FDG PET reads did not reveal any distinct pathological finding, including possible alternative diagnoses, in any of the patients. We also analyzed the PES of the previously established COVID-19-related metabolic covariance pattern. However, while this pattern tightly correlated to MoCA performance and was still elevated at trend level compared to control patients at follow-up in our studies in COVID-19 inpatients (at the subacute stage and 6 months later) $(8,9)$, none of the patients with Long COVID-syndrome exhibited this pattern. Notably, this also includes 4/14 patients showing impaired performance on MoCA who underwent ${ }^{18} \mathrm{~F}-\mathrm{FDG}$ PET. To exclude the possibility that the COVID-19-related metabolic covariance pattern established in subacute inpatients is simply not appropriate for patients with Long COVID-syndrome, we also conducted a conventional SPM group analysis, which, again, showed no pathological finding. Even for a subgroup of patients with abnormal MoCA scores $(n=4)$, we did not find any relevant differences in glucose metabolism compared to the remaining patients or the control cohort (voxel-wise SPM group analysis, exploratory threshold of $P<0.005$, data not shown). Although we cannot exclude the possibility that Long COVID-associated changes of neuronal activity are too subtle to be captured by an ${ }^{18}$ F-FDG PET group analysis, we consider this unlikely. Indeed, ${ }^{18} \mathrm{~F}$-FDG PET is a well-established marker of neuronal dysfunction for prodromal stages of neurological diseases of similar cognitive impact. Thus, together with the in large parts unimpaired cognitive battery across the entire group of patients, it appears reasonable that factors other than the cortical hypometabolism observed in patients 
during the early subacute stage after an infection (8) contribute to the symptoms in neurocognitive Long COVID-syndrome.

Our results have to be compared to other recent studies that employed cerebral ${ }^{18} \mathrm{~F}-\mathrm{FDG}$ PET for the assessment of COVID-19 associated metabolic changes. A frontal and, to a lesser extent, temporoparietal cortical hypometabolism, which improved during follow-up at 1 and 6 months, was detected by Kas and colleagues (10), which is in line with our observations $(8,9)$. Of note, different from our previous cohort $(8,9)$ patients included in the aforementioned study (10) suffered from COVID-19-related encephalopathy including delirium, seizures, myocloni, and focal neurological signs, whereas such severe symptoms were absent in the patients of our subacute cohort $(8,9)$. Clearly deviating from those studies and the present study, a profile of hypometabolism in limbic/paralimbic regions extending to the brainstem and cerebellum was reported for patients with putative Long COVID (including decreasing glucose metabolism of the right temporal lobe with longer time after first COVID-19 symptoms) (11). Factors like pooling of variable timepoints of examination (about 1-5 months after COVID-19, on average $96 \pm 31$ days) and the use of cortical regions for count rate normalization of PET data may have contributed to these discordant findings that are also contra-intuitive regarding recovery from COVIDrelated cognitive deficits in longitudinal investigations $(9,15)$ (for a detailed discussion see (32)). Sollini et al. (12) described a hypometabolism particularly of the right parahippocampal gyrus and thalamus in Long COVID patients examined at about 3-4 months after symptom onset. Again, technical factors may explain different findings (e.g., extraction of brain scans from whole-body examination, which may yield inferior data quality if whole-body PET acquisition parameters are not matched to brain acquisitions; 
retrospective use of brain images of oncological patients as control data, which limits standardization of behavior/sensory input during ${ }^{18} \mathrm{~F}-\mathrm{FDG}$ uptake; liberal statistical thresholds such as $P<0.005$ on voxel-level). Finally, a thorough qualitative and quantitative assessment of cognitive profiles and correlation to changes in cerebral glucose metabolism was not pursued by other studies (10-12), which underlines the particular value of the present work.

The lack of significant findings on ${ }^{18} \mathrm{~F}-\mathrm{FDG}$ PET and only mild impairments on neuropsychological testing is in contrast to the severe and lasting disability reported by the patients (e.g., cognitive symptoms, inability to work). Moreover, neither MoCA performance nor the composite z-score of the neurocognitive test battery correlated with disability. On the other hand and in line with other reports in Long COVID $(33,34)$, fatigue was particularly prevalent in our cohort (61\%, WEIMuS sumscore). Fatigue is a common sequel of systemic viral infections $(35,36)$, systemic inflammatory diseases (37) and has been related to immune dysregulation processes $(38,39)$ as in the systemic inflammatory response and cytokine release (14) in COVID-19. Fatigue has also been linked to the myalgic encephalomyelitis/chronic fatigue syndrome (ME/CFS (5)) in Long COVID, which is characterized by functional impairment (e.g., disability to work) in a considerable number of patients (40). Taken together, it is tempting to speculate that the pathophysiological background of self-reported cognitive symptoms, disability and even mild impairments in the neuropsychological test battery in single patients is primarily caused by fatigue.

As a limitation of the present study, only patients self-presenting with long-lasting symptoms were included in our cohort, thereby potentially presenting a small subgroup 
of COVID-19 patients. However, deficits reported in our cohort are corroborated by the rate of previously reported deficits $(2,3)$ and the fraction of patients that were still unable to work three to four months after infection reported previously (2) is in accordance with our cohort (i.e., $32 \%$ vs. $39 \%$ ). As an inherent problem of studies like ours, no data is available concerning the premorbid cognitive and neuropsychological status of the patients. Thus, we cannot comment on a possible particular vulnerability or pre-existing deficits, which is of particular interest if the detected impairment is small and inconsistent. Furthermore, the number of subjects is relatively small which precluded in-depth multivariate statistical analyses and limits the possibility to make reliable statements about the frequency of cognitive deficits in Long COVID patients. For instance, in contrast to the general observation that male sex is a risk factor for severe disease courses in COVID-19 (41), female sex seems to be slightly overrepresented in the present study. Longitudinal studies are needed to define the prognosis of neurocognitive symptoms in patients with Long COVID-syndrome. In this regard, the lack of long-lasting alterations of cerebral functioning on ${ }^{18} \mathrm{~F}-\mathrm{FDG}$ PET would be compatible with a favorable outcome.

\section{Disclosures}

PTM received honoraries for lectures and consulting by GE and Philips. HU received honoraria for lectures from Bracco, Bayer, Union Chimique Belge (UCB) pharma, Eisai, and Stryker. No other potential conflicts of interest relevant to this article exist.

\section{Funding}

No specific funding was received for this study 


\section{Data availability}

The data that support the findings of this study are available on request from the corresponding author. The data are not publicly available due to their containing information that could compromise the privacy of research participants.

\section{ACKNOWLEDGMENTS}

The use in this work of ScAnVP software, copyright @ 2020 The Feinstein Institute for

Medical Research, is hereby acknowledged. NS and AD were supported by the BertaOttenstein-Program for Clinician Scientists, Faculty of Medicine, University of Freiburg. 


\section{KEY POINTS}

QUESTION: Long-term neurocognitive symptoms after COVID-19 are an increasingly recognized problem - is the underlying pathophysiology a residual state of cortical dysfunction detected in subacute patients?

PERTINENT FINDINGS: In patients $(n=31)$ in the long-term phase after COVID-19 (202 \pm 58 days after positive PCR) with self-reported symptoms of Long COVID, an exhaustive neuropsychological test-battery revealed slight impairments only in individual cases, whereas fatigue was highly prevalent. Cerebral ${ }^{18}$ F-FDG PET failed to reveal a distinct pathological signature in the subgroup of patients undergoing ${ }^{18}$ F-FDG PET $(n=14)$.

IMPLICATIONS FOR PATIENT CARE: The findings deviate from previous reports in patients in the early subacute stage of COVID-19 and suggest that underlying causes of Long COVID might be related to fatigue but not to persistent cortical dysfunction. 


\section{REFERENCES}

1. Honigsbaum M, Krishnan L. Taking pandemic sequelae seriously: from the Russian influenza to COVID-19 long-haulers. The Lancet. 2020;396:1389-1391.

2. Garrigues E, Janvier P, Kherabi $Y$, et al. Post-discharge persistent symptoms and healthrelated quality of life after hospitalization for COVID-19. J Infect. 2020:e4-e6.

3. Halpin SJ, Mclvor C, Whyatt G, et al. Postdischarge symptoms and rehabilitation needs in survivors of COVID-19 infection: A cross-sectional evaluation. J Med Virol. 2020:10131022.

4. Woo MS, Malsy J, Pöttgen J, et al. Frequent neurocognitive deficits after recovery from mild COVID-19. Brain Commun. 2020;2(2):fcaa205.

5. Wildwing T, Holt N. Similarities between the neurological symptoms of COVID-19 and Functional Neurological Disorder: A systematic overview of systematic reviews and implications for future neurological healthcare services. Ther Adv Chronic Dis. 2021;Epub ahead of print.

6. Davis HE, Assaf GS, McCorkell L, et al. Characterizing long COVID in an international cohort: 7 months of symptoms and their impact. EClinicalMedicine. 2021;Epub ahead of print.

7. NICE guideline [NG188], National Institute for Health and Care Excellence. COVID-19 rapid guideline: managing the long-term effects of COVID-19. December 2020.

8. Hosp JA, Dressing A, Blazhenets G, et al. Cognitive impairment and altered cerebral glucose metabolism in the subacute stage of COVID-19. Brain. 2021;144(4):1263-1276.

9. Blazhenets G, Schröter N, Bormann T, et al. Slow but evident recovery from neocortical dysfunction and cognitive impairment in a series of chronic COVID-19 patients. J Nucl Med. 2021;62(7):910-915.

10. Kas A, Soret M, Pyatigoskaya N, et al. The cerebral network of COVID-19-related encephalopathy: a longitudinal voxel-based 18F-FDG-PET study. Eur J Nucl Med Mol Imaging. 2021;48(8):2543-2557.

11. Guedj E, Campion JY, Dudouet P, et al. 18F-FDG brain PET hypometabolism in patients with long COVID. Eur J Nucl Med Mol Imaging. 2021;48(2).

12. Sollini M, Morbelli S, Ciccarelli M, et al. Long COVID hallmarks on [18F]FDG-PET/CT: a case-control study. Eur J Nucl Med Mol Imaging. 2021;Epub ahead of print.

13. Matschke J, Lütgehetmann M, Hagel C, et al. Neuropathology of patients with COVID-19 in Germany: a post-mortem case series. Lancet Neurol. 2020;19:919-929.

14. Mehta P, McAuley DF, Brown M, Sanchez E, Tattersall RS, Manson JJ. COVID-19: consider cytokine storm syndromes and immunosuppression. The Lancet. 2020;395:10331034. 
15. Zhao S, Shibata K, Hellyer PJ, et al. Rapid vigilance and episodic memory decrements in COVID-19 survivors. preprint medRxiv; 2021.

16. COVID-19 Guidelines Robert Koch-Institute. Hinweise zu Erkennung, Diagnostik und Therapie von Patienten mit COVID-19. Robert-Koch Institut. December 2020.

17. Brandt J, Benedict R. Hopkins verbal learning test - Revised. Administration manual. Lutz, FL: Psychological Assessment Resources. 2001.

18. Benedict RHB, Schretlen D, Groninger L, Dobraski M, Shpritz B. Revision of the Brief Visuospatial Memory Test: Studies of normal performance, reliability, and validity. Psychological Assessment. 1996;8:145-153.

19. Lepach A, Petermann F. Wechsler Memory Scale - revised. Göttingen: Hogrefe; 2012.

20. Lezak MD. Neuropsychological assessment. 3rd ed. New York: Oxford University Press; 1995.

21. Bäumler, G. Farbe-Wort-Interferenztest (FWIT) nach J.R. Stroop. Göttingen: Hogrefe; 1985.

22. Langdon DW, Amato MP, Boringa J, et al. Recommendations for a Brief International Cognitive Assessment for Multiple Sclerosis (BICAMS). Mult Scler. 2012;18:891-898.

23. Aebi C, Monsch AU, Berres M, Brubacher D, Staehelin HB. Validation of the German CERAD-neuropsychological assessment battery. Neurobiol of Aging. 2002;23(1):27-28.

24. Lazar RM, Pavol MA, Bormann T, et al. Neurocognition and Cerebral Lesion Burden in High-Risk Patients Before Undergoing Transcatheter Aortic Valve Replacement. JACC Cardiovasc Interv . 2018;11:384-392.

25. Nasreddine ZS, Phillips NA, Bédirian V, et al. The Montreal Cognitive Assessment, MoCA: a brief screening tool for mild cognitive impairment. J Am Geriatr Soc. 2005;53:695-699.

26. Flachenecker P, Müller G, König H, Meissner H, Toyka KV, Rieckmann P. "Fatigue" in multiple sclerosis. Development and validation of the "Würzburger Fatigue Inventory for MS." Nervenarzt. 2006;77:165-166, 168-170, 172-174.

27. Yesavage JA, Sheikh JI. 9/Geriatric Depression Scale (GDS): Recent Evidence and Development of a Shorter Version. Clinical Gerontologist. 1986;5:165-173.

28. Minoshima S, Frey KA, Koeppe RA, Foster NL, Kuhl DE. A diagnostic approach in Alzheimer's disease using three-dimensional stereotactic surface projections of fluorine18-FDG PET. J Nucl Med. 1995;36:1238-1248.

29. Voruz P, Allali G, Benzakour L, et al. Long COVID neuropsychological deficits after severe, moderate or mild infection. preprint medRxiv; 2021.

30. Baddeley AD, Eysenck MW, Anderson MC. Memory. London: Routledge; 2020. 
31. Almeria M, Cejudo JC, Sotoca J, Deus J, Krupinski J. Cognitive profile following COVID-19 infection: Clinical predictors leading to neuropsychological impairment. Brain Behav Immun Health . 2020;9:100163.

32. Meyer PT, Blazhenets G, Prinz M, Hosp JA. Reply: From early limbic inflammation to long COVID sequelae. Brain. 2021:Epub ahead of print.

33. Townsend L, Dyer AH, Jones $\mathrm{K}$, et al. Persistent fatigue following SARS-CoV-2 infection is common and independent of severity of initial infection. Madeddu G, ed. PLoS ONE. 2020;15:e0240784.

34. Kedor C, Freitag H, Meyer-Arndt L, et al. Chronic COVID-19 Syndrome and Chronic Fatigue Syndrome (ME/CFS) following the first pandemic wave in Germany - a first analysis of a prospective observational study. medRxiv. 2021.

35. Moldofsky H, Patcai J. Chronic widespread musculoskeletal pain, fatigue, depression and disordered sleep in chronic post-SARS syndrome; a case-controlled study. BMC Neurology. 2011;11:37.

36. Islam MF, Cotler J, Jason LA. Post-viral fatigue and COVID-19: lessons from past epidemics. Fatigue: Biomedicine, Health \& Behavior. 2020;8(2):61-69.

37. Zielinski MR, Systrom DM, Rose NR. Fatigue, Sleep, and Autoimmune and Related Disorders. Front Immunol. 2019;10:1827.

38. Sotzny F, Blanco J, Capelli E, et al. Myalgic Encephalomyelitis/Chronic Fatigue Syndrome - Evidence for an autoimmune disease. Autoimmun Rev. 2018;17:601-609.

39. Komaroff A, Cho T. Role of Infection and Neurologic Dysfunction in Chronic Fatigue Syndrome. Semin Neurol. 2011;31:325-337.

40. Vink M, Vink-Niese F. Work Rehabilitation and Medical Retirement for Myalgic Encephalomyelitis/Chronic Fatigue Syndrome Patients. A Review and Appraisal of Diagnostic Strategies. Diagnostics (Basel). 2019;9(4):124.

41. Weiss $P$, Murdoch DR. Clinical course and mortality risk of severe COVID-19. Lancet. 2020;395:1014-1015. 


\section{Figures}

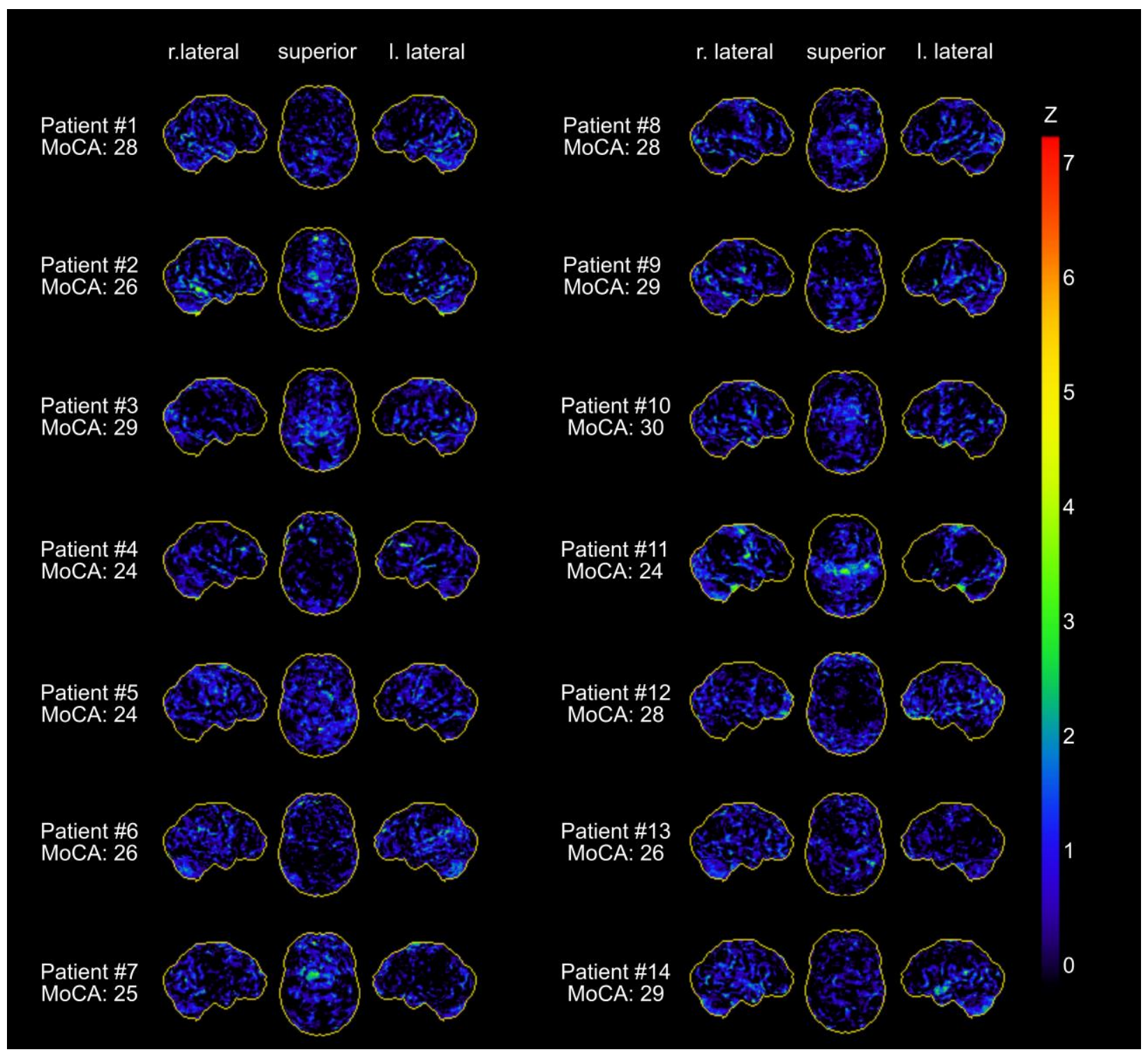

Figure 1. Individual results of voxelwise statistical analysis of ${ }^{18} \mathrm{~F}-\mathrm{FDG}$ PET data with NeuroSTAT/3D-SSP. Shown are lateral and superior views of the brain. Metabolic deficits compared with age-matched control subjects are color-coded as z scores. MoCA, Montreal cognitive assessment. Z, Z-score. 


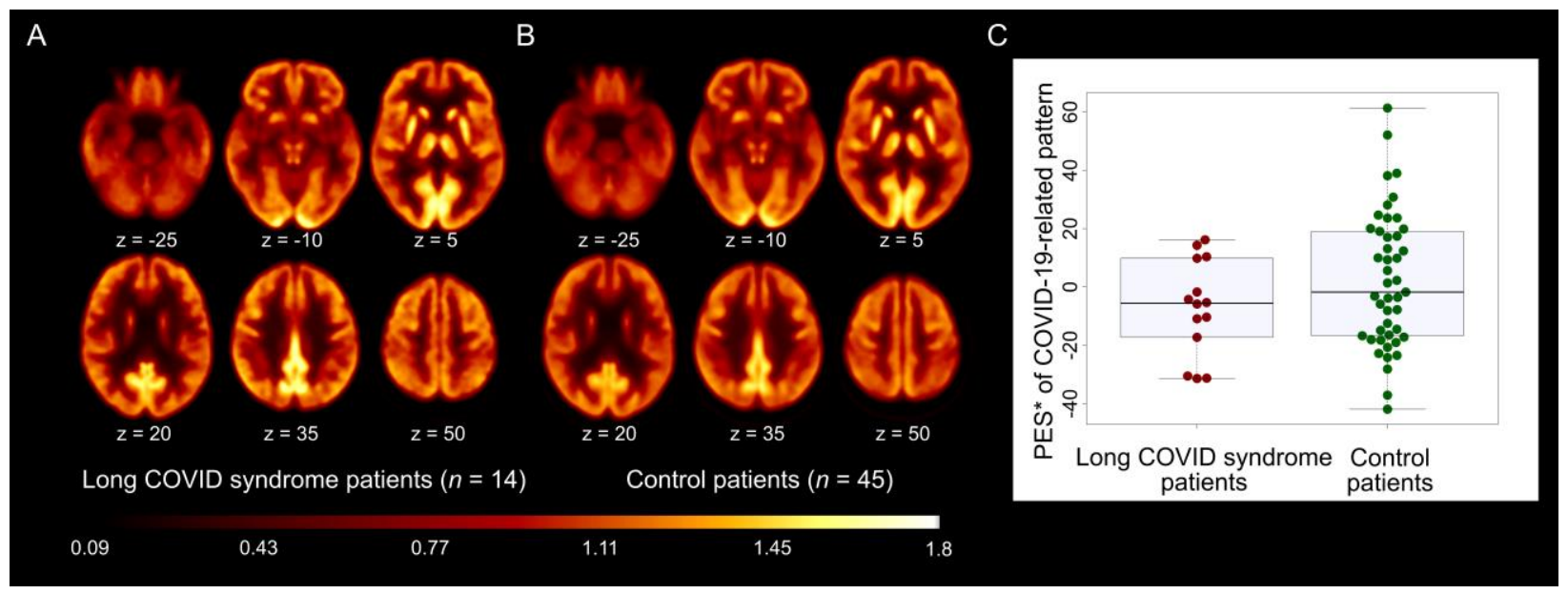

Figure 2. ${ }^{18} \mathrm{~F}-\mathrm{FDG}$ PET in patients with Long COVID-syndrome. A and B: Transaxial sections of group averaged, spatially normalized ${ }^{18} \mathrm{~F}-\mathrm{FDG}$ PET scans in patients with Long COVID-syndrome (A) and control patients (B). C: The pattern expression score (PES; *adjusted for age and sex, for illustration purposes) of the previously established COVID-19-related spatial covariance pattern was not significantly different between patients with Long COVID-syndrome and control patients. Boxplots (grey), as well as individual values for COVID-19 patients (red) and the control cohort (green), are displayed. 